\title{
Effect of Dynamic High-pressure Homogenization Conditions on Dietary Fiber from Mango Peel
}

\author{
Shao-dan Peng ${ }^{1, a}$, Xiao-bing Huang ${ }^{1, b,}$, Ji-hua Li ${ }^{1, c}$, Li-jing Lin ${ }^{1, d}$, Wen Xia ${ }^{1, e}$ \\ ${ }^{1}$ Agricultural Products Processing Research Institute, Chinese Academy of Tropical Agricultural \\ Sciences, Zhanjiang 524001, China

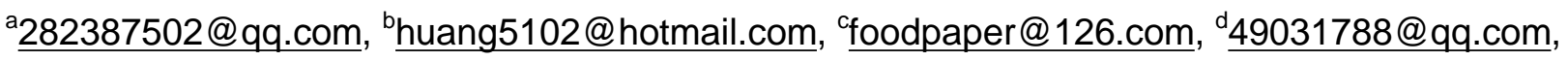 \\ exiawensg@163.com
}

Keywords: Mango peel, dietary fiber, dynamic high-pressure homogenization

Abstract. Dietary fiber obtained from mango peel (MPDF) by fermentation with lactic acid bacteria and ultrafine grinding was treated by dynamic high-pressure homogenization (DHPH). And the effect of DHPH conditions on composition and physicochemical properties of mango peel dietary fiber was investigated by determining the content of insoluble dietary fiber (IDF), soluble dietary fiber (SDF), cellulose, hemicellulose, lignin, water absorption capacity (WAC) and oil absorption capacity $(\mathrm{OAC})$. It was found that DHPH could increase the content of SDF in mango peel from $7.80 \%$ to $15.69 \%$, while the content of IDF decreased significantly, and the content of total dietary fiber showed a slight downward trend. The loss of hemicellulose was observed after DHPH, while the cellulose and lignin content did not show significant differences. In addition, there was a reduction of WAC and OAC in the treatment. The results indicated that DHPH would effectively promote the transformation of IDF to SDF in MPDF, which may be caused by the degradation of insoluble hemicellulose during DHPH.

\section{Introduction}

Mango (Mangifera Indica L.), belonging to the family Anacardiaceae, is the most important commercial fruit in tropical and subtropical regions, especially in China, India, Thailand, Pakistan, Burma, and so on. It is well known as the king of tropical fruit because of its micronutrients, vitamins, dietary fiber, carbohydrates, proteins, and other phytochemicals [1], which are vital to normal human growth, development and health [2]. As a seasonal fruit, mango fruits are usually processed into various products such as puree, beverages, nectar, leather, pickles, canned slices, etc., which have worldwide popularity [3]. During processing of mango, huge amount of peel is generated, it is a waste by-product, and its disposal is a major problem and causes environmental pollution. Mango peel, accounting for $15-20 \%$ of mango fruit, contains a number of valuable compounds such as polyphenols, carotenoids, enzymes and dietary fibers (Ajila et al., 2007a). Especially, it was proved that nearly $54 \%$ of the total dietary fiber (TDF) content in dried mango peel [4].

Dietary fiber (DF), a group of health-promoting food components consisting of polysaccharides, oligosaccharides and lignin[5], can be divided into soluble dietary fiber (SDF) and insoluble dietary fiber (IDF) according to the solubleness of dietary fiber in water. It has been reported that sufficient intake of DF is beneficial to normal gastrointestinal and physiological functions, including a reduced risk of coronary heart disease, diabetes, obesity, and some cancers [6]. The health benefits of dietary fiber have led to increased consumption of DF-rich products. By-products from the fruit 
and vegetable are good sources of dietary fiber, since they are cheap and available in large quantity. Generally, IDF was the predominant fraction in dietary fiber of by-products in many fruits and vegetables such as mango and carrot peel $[4,5]$. However, as a potential prebiotic character, SDF plays more important role in the improvement of gastroenteric environments and glucose tolerance in diabetes than IDF does [7]. Unremitting efforts have been made to modify the IDFs from different sources using different techniques, such as fermentation, enzymatic, chemical or physical treatments [8-11]. Among these methods, dynamic high pressure homogenization (DHPH) treatment has become a promising homogenization technology for biomass refining, due to its simplicity, high efficiency and the lack of a requirement for organic solvents [12]. However, it was merely used in the modification of IDF from soybean residue and cellulose from bagasse [13-15], while few studies have been reported to focus on the modification of dietary fiber from fruit bypoucts such as mango peel using DHPH method. The present study attempted to investigate how DHPH could affect the dietary fibre in mango peel, which was characterized by the content, composition, WAC and OAC of dietary fiber.

\section{Materials and methods}

Preparation of MPDF. Peel of Ripe Australian mango planting in Hainan province of China was collected and processed into DF powder referring to the fermentation methods by Huang et al [4]. Then the MPDF were obtained by ultrafine grinding the powders for $15 \mathrm{~min}$ in a WZJ6 micronizing pharmaceutical vibrating mill (Jinan Billion Powder Engineering Co., LTD, China).

DHPH. Dynamic high pressure homogenization experiments were performed on laboratory scale with a high pressure homogenizer (AH100D, ATS Engineering Inc., Canada). Specifically, the MPDF powders were dispersed in water with concentrations of $2 \%, 4 \%, 6 \%, 8 \%$ and $10 \%$ and stirred gently at $25^{\circ} \mathrm{C}$ for $30 \mathrm{~min}$. Then the MPDF suspension was homogenized at pressure levels ranging from 30 to $150 \mathrm{Mpa}$, while the different DHPH passes from 5 to 25 cycles were also investigated. Finally, the solutions were collected and freeze-dried in a vacuum freeze drying equipment (Alpha 2-4 Ldplus, CHRIST, Germany) until reaching a constant weight.

Fiber Analysis. The Insoluble dietary fiber (IDF), soluble dietary fiber (SDF), and total dietary fibre (TDF) were determined using the Megazyme TDF Test Kit (K-TDFR, Megazyme International Ireland, Bray Business Park, Bray, Co. Wicklow, Ireland.) exactly according to enzymatic-gravimetric method with MES-TRIS buffer, based on the AOAC991.43[16]. Acid detergent fiber (ADF) and acid detergent lignin (ADL) were determined using the AOAC method 973.18 [17], whereas the NDF was determined using the method reported by van Soest et al.[18].

Determination of WAC and OAC [4]. Two centrifuge tubes were weighed separately with individual weights. $1.000 \mathrm{~g}$ of sample was then put in each tube. $40 \mathrm{~mL}$ of distilled water and soybean oil were added and stirred with sample to avoid lumps in the water bath with $25^{\circ} \mathrm{C}$ for $24 \mathrm{~h}$, respectively. The tubes were centrifuged at $3500 \mathrm{rpm}$ for $30 \mathrm{~min}$. The water or oil was then decanted from the tube without loss of solids.Finally, the tube and residue were weighed out. The WAC or OAC was calculated as following equation:

WAC or OAC $(\%)=\frac{\text { Weight of centrifuge tube and sample }- \text { Centrifuge tube }}{\text { Weight of sample }} \times 100 \mathrm{~L} L$ (1)

Statistical Analysis. All experiments were performed in triplicate and the datas were presented as mean \pm standard deviation. The data processing was carried out using ORIGIN software (Version 8.6, OriginLab Corporation) and SPSS Statistics (Version 20, IBM). 


\section{Results and discussion}

Effect of Concentration on MPDF. MPDF was enriched in dietary fibre (68.00\%), and IDF was the main fibre fraction (60.20\%). The ratio of insoluble to soluble dietary fibre in MPDF was 7.70. TDF content in MPDF was higher than those reported previously by Ajila et al. (51.20\%) [19], which was potentially attributed to the different procesing methods[4], cultivars and maturity [20]. The contents of cellulose, hemicellulose and lignin in MPDF were determine as $18.38 \%, 24.60 \%$, $10.5 \%$, respectively. The effects of different MPDF concentrations under DHPM processing on fiber composition, WAC and OAC of MPDF were presented in Fig. 1a-c.

As shown in Fig. 1a, the concentrations of MPDF in the solution to be homogenized didn't affect TDF content, but it made SDF increase and IDF decrease significantly, and both varied with the differing concentrations. About $35.41 \%$ of reduction was apparently observed in hemicellulose content during DHPH, while the cellulose content decreased by $20.84 \%$ followed by $18.71 \%$ of the lignin reduction as presented in Fig. 1b. From Fig. 1c we can see that the WAC and OAC presented a downward trend with the increase of MPDF concentration, which was possibly due to that the cellulose and hemicellulose in MPDF degraded into soluble monosaccharides in the DHPH processing.

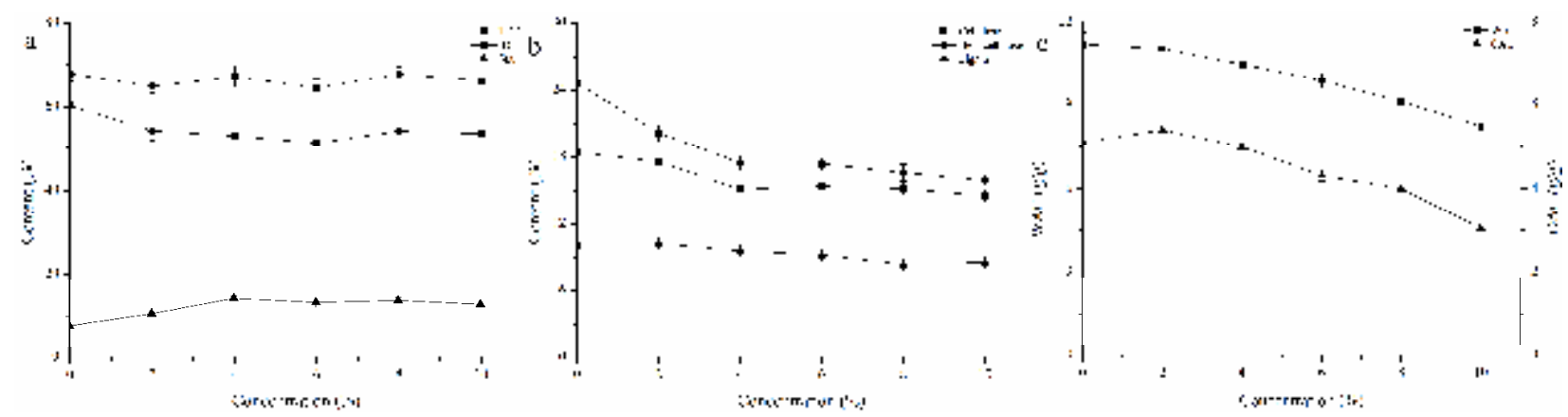

Fig. 1 Effect of concentration on dietary fiber content (a), fiber composition (b), WAC and OAC (c) in MPDF

Effect of DHPH Pressure on MPDF. The MPDF were homogenized under pressures from 30 to $150 \mathrm{Mpa}$, and there was a significant increase in SDF $(7.80 \%$ to $15.69 \%)$ content as shown in Fig. $2 \mathrm{a}$. On the contrary, the IDF content dropped significantly from $60.20 \%$ to $51.06 \%$. And the ratio of insoluble to soluble dietary fibre was decreased from 7.70 to 3.25 , while no significant difference the TDF content. The content of dietary fibre's fractions in MPDF were determined to demonstrate whether cellulose, hemicellulose or lignin partially degraded into soluble substances (Fig. 2b). The hemicellulose degradation under DHPH may account for the SDF increase and IDF reduction in line with the research by Tu et al. Zia ur et al.[14, 21], which possibly led to the decline of WAC and OAC in Fig. 2c.

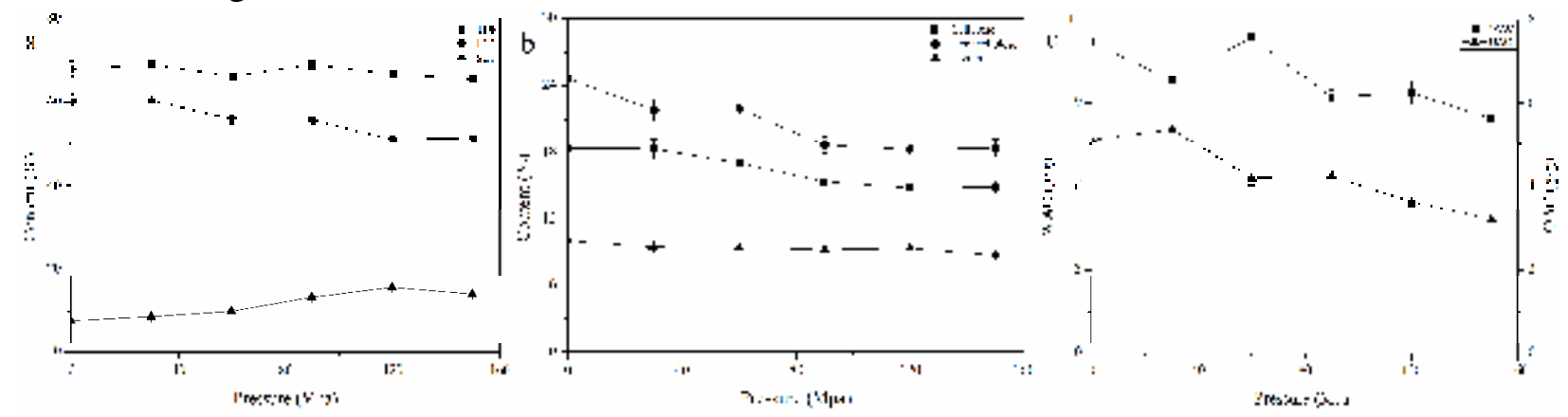

Fig. 2 Effect of DHPH pressure on dietary fiber content (a), fiber composition (b), WAC and OAC (c) in MPDF

Effect of DHPH Cycles on MPDF. The effect of DHPH cycles on MPDF was presented in Fig. 
3a-c. With the increase of DHPH cycles, the increasing trend of SDF content and the decreasing trend of IDF content were almost the same as those with pressure increasing (Fig. 3a). DHPM treatment did not affect the amount of cellulose, but affected hemicellulose content significantly. During DHPH from 10 to 20 cycles, the hemicellulose content declined rapidly to the lowest value as shown in Fig. 3b. A little decrease was found in WAC and OAC of MPDF after DHPH (Fig. 3c), which may be caused by physical damage of the loose and porous fiber structure generated in the fermentation preparation during DHPH course.

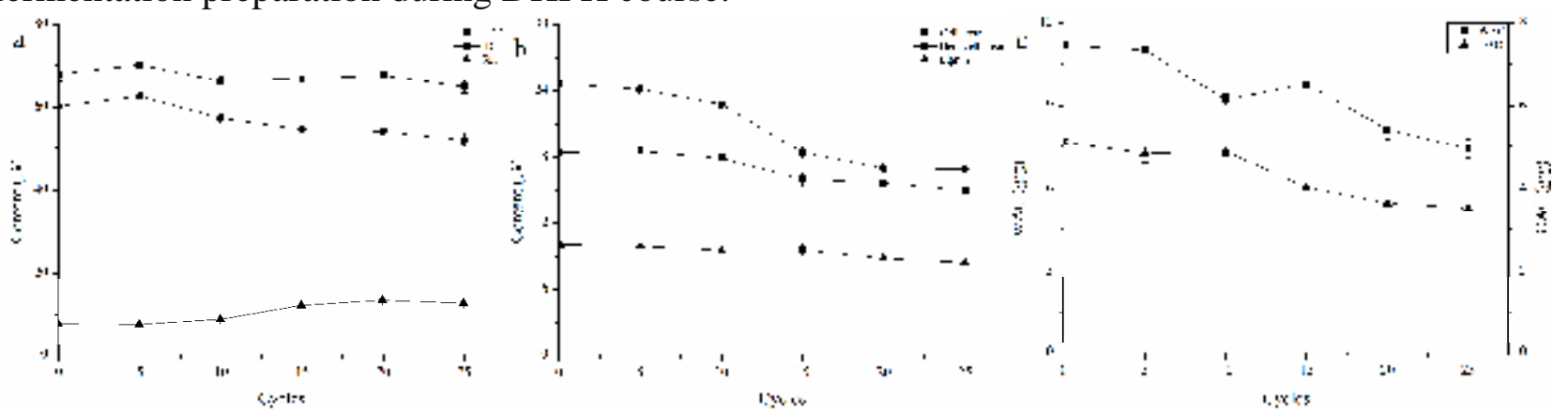

Fig. 3 Effect of DHPH cycles on dietary fiber content (a), fiber composition (b), WAC and OAC (c) in MPDF

\section{Conclusions}

The DHPH treatment could help improving the composition of the mango peel dietary fiber. The SDF content was in increased while the IDF content reduced significantly, and the hemicellulose was preferentially solubilized during DHPH pocessing. The combined forces of instantaneous pressure drop, intense shear, cavitation and ultra-high pressures caused by DHPH could bring to the breakage of weak bonds between polysaccharides, which may result in the increasing SDF content. The deep-seated mechanism should be verified through more additional technologies. Nevertheless, DHPH could make mango peel dietary fiber give more SDF and may be used as a promising methods to improve the quality of dietary fibre from fruit byproducts.

\section{Acknowledgements}

This research was supported by the Province Natural Science Foundation of Hainan (Project No. 20163114 and No. 20163113) and the Special Scientific Research Fund of Agricultural Public Welfare Profession of China (Grant No. 201503142-14).

\section{References}

[1] R. Tharanathan, H. Yashoda, T. Prabha: Mango (Mangifera indica L.),"The king of fruits"-An overview, Food Reviews International, 22 (2006), p. 95.

[2] M. Jahurul, I. Zaidul, K. Ghafoor, F.Y. Al-Juhaimi, K.-L. Nyam, N. Norulaini, F. Sahena, A.M. Omar: Mango (Mangifera indica L.) by-products and their valuable components: A review, Food chemistry, 183 (2015), p. 173.

[3] D. Loeillet: The European mango market: A promising tropical fruit, Fruits, 49 (1994), p. 434.

[4] X. Huang, S. Peng, J. Li, M. Li, K. Liu, X. Gong, L. Lin: Study on the preparation process of dietary fi ber from mango peel by fermentation, Science and Technology of Food Industry, (2017), p. 153.

[5] P. Chantaro, S. Devahastin, N. Chiewchan: Production of antioxidant high dietary fiber powder from carrot peels, LWT-Food Science and Technology, 41 (2008), p. 1987. 
[6] J. Mann, J. Cummings: Possible implications for health of the different definitions of dietary fibre, Nutrition, Metabolism and Cardiovascular Diseases, 19 (2009), p. 226.

[7] N. Grigelmo-Miguel, O. Martín-Belloso: Comparison of dietary fibre from by-products of processing fruits and greens and from cereals, LWT-Food Science and Technology, 32 (1999), p. 503.

[8] A. Napolitano, S. Lanzuise, M. Ruocco, G. Arlotti, R. Ranieri, S.H. Knutsen, M. Lorito, V. Fogliano: Treatment of cereal products with a tailored preparation of Trichoderma enzymes increases the amount of soluble dietary fiber, Journal of agricultural and food chemistry, 54 (2006), p. 7863.

[9] A.M. Lambo, R. Öste, M.E.-L. Nyman: Dietary fibre in fermented oat and barley $\beta$-glucan rich concentrates, Food chemistry, 89 (2005), p. 283.

[10] A. Sangnark, A. Noomhorm: Chemical, physical and baking properties of dietary fiber prepared from rice straw, Food Research International, 37 (2004), p. 66.

[11] S. Raghavendra, S.R. Swamy, N. Rastogi, K. Raghavarao, S. Kumar, R. Tharanathan: Grinding characteristics and hydration properties of coconut residue: A source of dietary fiber, Journal of Food Engineering, 72 (2006), p. 281.

[12] M. Keerati-u-rai, M. Corredig: Effect of dynamic high pressure homogenization on the aggregation state of soy protein, Journal of agricultural and food chemistry, 57 (2009), p. 3556.

[13] C.-m. Liu, R.-h. Liang, T.-t. Dai, J.-p. Ye, Z.-c. Zeng, S.-j. Luo, J. Chen: Effect of dynamic high pressure microfluidization modified insoluble dietary fiber on gelatinization and rheology of rice starch, Food Hydrocolloids, 57 (2016), p. 55.

[14] Z. Tu, L. Chen, H. Wang, C. Ruan, L. Zhang, Y. Kou: Effect of fermentation and dynamic high pressure microfluidization on dietary fibre of soybean residue, Journal of food science and technology, (2014), p. 1.

[15] J. Li, X. Wei, Q. Wang, J. Chen, G. Chang, L. Kong, J. Su, Y. Liu: Homogeneous isolation of nanocellulose from sugarcane bagasse by high pressure homogenization, Carbohydrate polymers, 90 (2012), p. 1609.

[16] W. Horwitz: Total, soluble and insoluble dietary fiber in foods, AOAC Official Methods of Analysis, 16th edition., Arlington, Virginia AOAC, (1995), p. 7.

[17] AOAC: Fiber (acid detergent) and lignin in animal feeds, Official method of analysis of the Association of Official Analytical Chemists, Arlington, VA: Association of Official Analytical Chemists, 1990.

[18] P.v. Van Soest, J. Robertson, B. Lewis: Methods for dietary fiber, neutral detergent fiber, and nonstarch polysaccharides in relation to animal nutrition, J. Dairy Sci., 74 (1991), p. 3583.

[19] C. Ajila, M. Aalami, K. Leelavathi, U.P. Rao: Mango peel powder: A potential source of antioxidant and dietary fiber in macaroni preparations, Innovative Food Science \& Emerging Technologies, 11 (2010), p. 219.

[20] C.M. Ajila, U.J.S. Prasada Rao: Mango peel dietary fibre: Composition and associated bound phenolics, Journal of Functional Foods, 5 (2013), p. 444.

[21] Z. Zia-ur-Rehman, M. Islam, W. Shah: Effect of microwave and conventional cooking on insoluble dietary fibre components of vegetables, Food chemistry, 80 (2003), p. 237. 